\title{
Emerging Treatment Options for the Management of Metastatic Colorectal Cancer
}

\author{
Presented by Dustin A. Deming, MD
}

\begin{abstract}
An improved understanding of the molecular landscape of metastatic colorectal cancer (CRC) has opened the door for new treatment options. Clinicians should test for molecular alterations that predict resistance to epidermal growth factor receptor (EGFR) inhibitors, such as KRAS and NRAS, and additionally clinically actionable alterations, including BRAFV600 mutations, HER2 amplification, NTRK fusions, and mismatch repair deficiency. Improved outcomes can be achieved with precision treatment strategies for the various CRC subtypes, although clinical features, such as tumor bulk and patient performance status, still help to guide treatment choice. Immune therapies have also produced impressive results in patients with mismatch repair-deficient/microsatellite instability-high tumors. These newer approaches were recently incorporated into the NCCN Guidelines for Colon and Rectal Cancers. In the future, these newer approaches may be used in earlier treatment settings.

J Natl Compr Canc Netw 2020;18(7.5):949-952
\end{abstract} doi: 10.6004 /jnccn.2020.5016

The treatment paradigm for metastatic colorectal cancer (CRC) has significantly changed in recent years. "It's no longer just FOLFOX and FOLFIRI," said Dustin A. Deming, $\mathrm{MD}$, Associate Professor, Carbone Cancer Center at the University of Wisconsin, during his presentation on emerging treatment options for this malignancy at the NCCN 2020 Virtual Annual Conference.

Historically, for virtually all patients with metastatic $\mathrm{CRC}$, treatment was initiated with cytotoxic chemotherapy independent of any molecular features of the tumor. Across the continuum of treatment, patients usually received FOLFOX (5-FU/leucovorin/oxaliplatin) and/or FOLFIRI (5-FU/leucovorin/irinotecan) \pm bevacizumab and an agent targeting the epidermal growth factor receptor (EGFR). Salvage regimens included regorafenib, tipiracil, and trifluridine.

An improved understanding of the molecular profile of CRC made it clear, however, that "this is not one disease, and the molecular subtypes of CRC are important in guiding treatment strategies," Dr. Deming said. Although many patients still receive conventional treatments in the first line, "for many patients we should think about other regimens, both in the first-line setting and potentially in the treatment-refractory setting," he said. Examples are FOLFOXIRI (5-FU/leucovorin/oxaliplatin/ irinotecan) + bevacizumab in earlier lines and, for patients deemed unsuitable for intensive cytotoxic chemotherapy, more targeted therapies and immunotherapies in some subsets of patients.
New treatment approaches for CRC take into account patient, clinical, and molecular features. "We need to know what mutations are present-which subtype of colon cancer we're dealing with-because that will help us tailor better treatment strategies."

It is now standard to test all metastatic tumors for KRAS, NRAS, and BRAF V600 mutations, HER2 amplification, and microsatellite instability (MSI) or mismatch repair deficiency (dMMR). Many patients are also tested for NTRK fusions. Updates to the NCCN Clinical Practice Guidelines in Oncology (NCCN Guidelines) for Colon and Rectal Cancers reflect the importance of these alterations and their targeted treatment.

\section{dMMR/MSI-High Metastatic CRC}

dMMR/MSI-high (MSI-H) cancers are a heterogeneous and rare subgroup, constituting approximately $3 \%$ of metastatic CRCs. ${ }^{1}$ Testing for dMMR/MSI-H status can be performed easily and reliably by immunohistochemistry, which identifies common alterations and is standard of care in many centers. An alternative, the PCR test, is a stand-alone functional assay that is well-validated.

Increasingly, however, clinicians are opting to utilize next-generation sequencing panels to evaluate MSI status. These tests not only detect microsatellite alterations but can also provide information about the actual mutations occurring in the $M M R$ genes. The drawback is that the validation of these tests is less robust than for the PCR microsatellite test. 
$\mathrm{dMMR} / \mathrm{MSI}-\mathrm{H}$ tumors can have alterations in different $M M R$ genes or proteins, can be germline or somatic, and can harbor "hundreds if not thousands" of mutations and fusions. "We think it's the number of these alterations that gives rise to increased neoantigen load and potentially increases the benefit from immunebased therapy," Dr. Deming said.

Several studies have documented encouraging outcomes in patients with treatment-refractory $\mathrm{dMMR} / \mathrm{MSI}-\mathrm{H}$ tumors treated with checkpoint inhibitors, including KEYNOTE164 (single-agent pembrolizumab) ${ }^{2}$ and CheckMate-142 (single-agent nivolumab and nivolumab + ipilimumab). ${ }^{3}$ Agents targeting PD-1 have become standard of care for patients with dMMR/MSI-H tumors.

"We may also be able to use these new therapies in the first-line setting," he predicted. In a cohort of patients from the CheckMate-142 trial, upfront use of nivolumab/ ipilimumab led to tumor reductions in $84 \%$ of patients and a 15-month progression-free survival (PFS) rate of $75 \% .{ }^{4}$ Single-agent pembrolizumab also produced a robust benefit in patients with dMMR/MSI-H tumors in KEYNOTE-177, ${ }^{5}$ with a median PFS of 16.5 months versus 8.2 months with treatment of physician's choice (hazard ratio, $0.60 ; P=.0002$ ). "These are exciting data that indicate that we should use immunotherapy options for patients in the first-line setting with dMMR cancers," Dr. Deming commented.

The phase III COMMIT trial is now evaluating modified FOLFOX6 + bevacizumab with or without the anti-PD-L1 agent atezolizumab versus atezolizumab alone in the first-line treatment of patients with dMMR tumors (ClinicalTrials.gov identifier: NCT02997228).

\section{BRAF V600-Mutant CRC}

$B R A F$ V600-mutated tumors are right-sided predominant and have larger primaries. They are associated with MMR deficiency, high-grade peritoneal disease, and metastatic lymph node involvement. Historically, targeting $B R A F$ became an exciting concept based on successes seen in melanoma. Although response rates to single-agent BRAF inhibitors in CRC are low, results of BRAF in combination with MEK or EGFR inhibitors have been more encouraging.

"Not all BRAF mutations are equal," he said. The V600E mutation, which occurs in $8 \%$ to $10 \%$ of patients with CRC, accounts for $80 \%$ of $B R A F$ mutations. Prognosis varies by mutation type: overall survival (OS) is poor for patients with V600E mutations, but for patients with non-V600E mutations, survival is comparable to those with $B R A F$ wild-type tumors. The NCCN Guidelines state that chemotherapy should be the first-line choice for $B R A F$ V600-mutated microsatellite-stable CRC. ${ }^{6} \mathrm{Al}-$ though FOLFOX and FOLFIRI are standard, there is emerging interest in using FOLFOXIRI + bevacizumab, especially for the more challenging V600E-mutant tumors.
In the TRIBE study, FOLFOXIRI/bevacizumab was associated with a median PFS of 7.5 months compared with 5.5 months for FOLFIRI/bevacizumab, and OS was 19.0 versus 10.7 months, respectively. ${ }^{7}$ Recently, TRIBE-2 found median PFS to be 12.0 versus 9.8 months, respectively. ${ }^{8}$

In the BEACON trial, ${ }^{9}$ approximately $50 \%$ of patients responded to the triplet regimen of encorafenib/ binimetinib/cetuximab, and median PFS was 8 months. These data led to an update to the NCCN Guidelines for patients with BRAF V600-mutated disease, although an update of that study concluded that binimetinib is not a necessary component. ${ }^{10}$ The doublet of encorafenib and either cetuximab or panitumumab is the new standard of care in this setting, yielding "dramatic improvements" over historical outcomes with second-line cytotoxic chemotherapy, according to Dr. Deming.

\section{HER2-Amplified RAS/RAF Wild-Type CRC}

HER2-amplified CRC, which is seen in approximately $5 \%$ of patients with metastatic disease, is a new subtype. Given promising treatment options for this subtype, the standard of care is to test all patients for HER2 amplification and overexpression. This can be performed with MMR immunohistochemistry, HER2 fluorescent in situ hybridization, and, increasingly, next-generation sequencing. Patients with $\geq 10$ HER 2 copy numbers are eligible for anti-HER2 agents, whereas those with levels $<8$ are not likely to benefit.

The NCCN Guidelines for Colon and Rectal Cancers were updated to reflect the recommendation of 2 firstline regimens for patients with HER2-amplified, RAS wild-type tumors deemed unsuitable for intensive chemotherapy: pertuzumab + trastuzumab and trastuzumab + lapatinib. These recommendations are based on the robust and durable responses seen in the MyPathway study $^{11}$ and the HERACLES study. ${ }^{12}$

"It's also reasonable to treat these patients with cytotoxic chemotherapy, and even with anti-EGFR agents, especially those with low-level amplification," Dr. Deming added. SWOG 1613 will compare trastuzumab/ pertuzumab with cetuximab/irinotecan in HER2amplified metastatic CRC in patients with treatmentrefractory disease and should help settle the issues of the optimal treatment of this population (ClinicalTrials.gov identifier: NCT03365882).

\section{RAS/RAF/HER2 Wild-Type Microsatellite-} Stable CRC

For "pan wild-type," microsatellite-stable tumors, treatment selection can be informed by the location of the primary tumor, based on data from CALGB $80405^{13}$ and FIRE-3. ${ }^{14}$ Patients with left-sided primary tumors are much more likely to have prolonged survival and are 
more likely to benefit from anti-EGFR therapy. Those with right-sided primaries are more likely to derive more benefit from bevacizumab than cetuximab in the firstline setting. "There are now data in the first-line and refractory settings that sidedness matters, and that antiEGFR therapy should be largely reserved for those patients who have wild-type KRAS, NRAS, and BRAF mutations and left-sided primaries," he said.

Dr. Deming and colleagues have shown that tumor bulk is important from the results of a study of 62 patients with KRAS wild-type tumors treated with anti-EGFR therapy. Patients with left-sided nonbulky tumors (no metastatic lesion $>3.5 \mathrm{~cm}$ ) had a median PFS of 7.2 months versus 3.5 months for the bulkier tumor cohort $(P=.001)$ and median OS of 23.4 versus 6.0 months $(P<.001) .{ }^{15}$ This suggests that patients with left-sided nonbulky wild-type disease may be best served with an anti-EGFR agent.

There is still debate regarding the best first-line regimen for RAS/ RAF/ HER2 wild-type metastatic CRC, and the NCCN Guidelines list many options, which are stratified by whether the patient is suitable for intensive therapy. "My practice is typically to use FOLFOX + bevacizumab for most patients, and there are some settings where I think about using an anti-EGFR agent in the first-line," Dr. Deming offered.

An attractive new concept for patients who respond to an anti-EGFR agent is to use anti-EGFR therapy again in the course of treatment. This paradigm would start with typical chemotherapy, moving to anti-EGFR as a single agent in the next line of therapy, followed by more chemotherapy in the next line, and re-treating with an antiEGFR drug after that. A number of studies have shown the potential for significant response in such patients. ${ }^{16,17}$

\section{NTRK Fusions}

NTRK fusions occur in $<0.5 \%$ of patients with metastatic CRC and are predictive of potential benefit to NTRK inhibitors, including larotrectinib and entrectinib. NTRK mutations, on the other hand, do not predict sensitivity, and these agents should not be prescribed for these patients.

Studies published in 2020 have confirmed that the "vast majority" of patients with NTRK fusions benefit from NTRK inhibitors. ${ }^{18,19}$ The question has thus become: Should all patients be tested?

"In CRC, given the rarity of this alteration, it doesn't make sense to test specifically for NTRK fusions, but I do favor testing for them as part of a larger panel. It's in the setting of testing for KRAS, NRAS, and BRAF that we could obtain this information," Dr. Deming said.

Disclosures: Dr. Deming has disclosed that he receives grant/research support from AbbVie, Inc., Bristol-Myers Squibb Company, Eli Lilly and Company, EMD Serono, Merck \& Co., Inc., and Revolution Machines; is a scientific advisor for Bayer HealthCare, Bristol-Myers Squibb Company, Genentech, Inc., Ipsen, Novocure, Array BioPharma, Promega, and Taiho Parmaceuticals Co., Ltd.; and receives consulting fees from Bayer HealthCare, Bristol-Myers Squibb Company, Genentech, Inc., Ipsen, Novocure, Array BioPharma, Promega, Taiho Parmaceuticals Co., Ltd.

Correspondence: Dustin A. Deming, MD, University of Wisconsin Carbone Cancer Center, 1111 Highland Avenue, WIMR11 6507, Madison, WI 53702. Email: ddeming@medicine.wisc.edu

\section{References}

1. Koopman M, Kortman GA, Mekenkamp L, et al. Deficient mismatch repair system in patients with sporadic advanced colorectal cancer. Br J Cancer 2009;100:266-273.

2. Le DT, Kim TW, Van Cutsem E, et al. Phase II open-label study of pembrolizumab in treatment-refractory, microsatellite instability-high/ mismatch repair-deficient metastatic colorectal cancer: KEYNOTE-164. J Clin Oncol 2020;38:11-19.

3. Overman MJ, Lonardi S, Wong KYM, et al. Durable clinical benefit with nivolumab plus ipilimumab in DNA mismatch repair-deficient/ microsatellite instability-high metastatic colorectal cancer. J Clin Oncol 2018;36:773-779

4. Lenz HJ, Lonardi S, Zagonel V, et al. Nivolumab plus low-dose ipilimumab as first-line therapy in microsatellite instability-high/DNA mismatch repairdeficient metastatic colorectal cancer: clinical update [abstract]. Presented at the ASCO 2020 Gastrointestinal Cancers Symposium; January 23-25, 2020; San Francisco, California. Abstract 11

5. Andre T, Shiu KK, Kim TW, et al. Pembrolizumab versus chemotherapy for microsatellite instability-high/mismatch repair deficient metastatic colorectal cancer: the phase 3 KEYNOTE-177 study [abstract]. Presented at the ASCO20 Virtual Scientific Program; May 29-31, 2020; Chicago, IIIlinois. Abstract LBA4.

6. Benson AB III, Venook AP, Al-Hawary MM, et al. NCCN Clinical Practice Guidelines in Oncology for Colon Cancer. Version 2.2020. Accessed March 1, 2020. Available at NCCN.org

7. Cremolini C, Loupakis F, Antoniotti C, et al. FOLFOXIRI plus bevacizumab versus FOLFIRI plus bevacizumab as first-line treatment of patients with metastatic colorectal cancer: updated overall survival and molecular subgroup analyses of the open-label, phase 3 TRIBE study. Lancet Oncol 2015;16:1306-1315.
8. Cremolini C, Antoniotti C, Lonardi S, et al. Updated results of TRIBE2, a phase III, randomized strategy study by GONO in the first- and second-line treatment of unresectable mCRC [abstract]. J Clin Oncol 2019;37(Suppl 15):Abstract 3508

9. Kopetz S, Grothey A, Yaeger R, et al. Encorafenib, binimetinib, and cetuximab in BRAF V600E-mutated colorectal cancer. N Engl J Med 2019 381:1632-1643

10. Kopetz S, Grothey A, Van Cutsem E, et al. Encorafenib plus cetuximab with or without binimetinib for BRAF V600E-mutant metastatic colorectal cancer: quality-of-life results from a randomized, three-arm, phase III study versus the choice of either irinotecan or FOLFIRI plus cetuximab (BEACON CRC) [abstract]. J Clin Oncol 2020;38(Suppl 1):Abstract 8.

11. Meric-Bernstam F, Hurwitz H, Raghav KPS, et al. Pertuzumab plus trastuzumab for HER2-amplified metastatic colorectal cancer (MyPathway): an updated report from a multicentre, open-label, phase $2 a$, multiple basket study. Lancet Oncol 2019;20:518-530.

12. Sartore-Bianchi A, Trusolino L, Martino C, et al. Dual-targeted therapy with trastuzumab and lapatinib in treatment-refractory, KRAS codon 12/13 wild-type, HER2-positive metastatic colorectal cancer (HERACLES): a proof-of-concept, multicentre, open-label, phase 2 trial. Lancet Oncol 2016;17:738-746.

13. Venook AP, Niedzwiecki D, Innocenti $F$, et al. Impact of primary tumor location on overall survival and progression-free survival in patients with metastatic colorectal cancer: analysis of CALGB/SWOG 80405 (Alliance) [abstract]. J Clin Oncol 2017;34(Suppl):Abstract 3504.

14. Tejpar S, Stintzing $S$, Ciardiello $F$, et al. Prognostic and predictive relevance of primary tumor location in patients with RAS wild-type metastatic colorectal cancer: retrospective analyses of the CRYSTAL and FIRE-3 trials. JAMA Oncol 2017;3:194-201. 
15. Kratz JD, Uboha NV, Lubner SJ, et al. Metastatic bulk independently predicts outcomes for EGFR precision targeting in colorectal cancer. J Natl Compr Canc Netw 2018;16:1442-1450.

16. Mauri G, Pizzutilo EG, Amatu A, et al. Retreatment with anti-EGFR monoclonal antibodies in metastatic colorectal cancer: systematic review of different strategies. Cancer Treat Rev 2019;73:41-53.

17. Liu X, George GC, Tsimberidou AM, et al. Retreatment with antiEGFR based therapies in metastatic colorectal cancer: impact of intervening time interval and prior anti-EGFR response. BMC Cancer 2015;15:713.

18. Doebele RC, Drilon A, Paz-Ares L, et al. Entrectinib in patients with advanced or metastatic NTRK fusion-positive solid tumours: integrated analysis of three phase 1-2 trials. Lancet Oncol 2020;21:271-282.

19. Hong DS, DuBois SG, Kummar S, et al. Larotrectinib in patients with TRK fusion-positive solid tumours: a pooled analysis of three phase $1 / 2$ clinica trials. Lancet Oncol 2020:21:531-540.

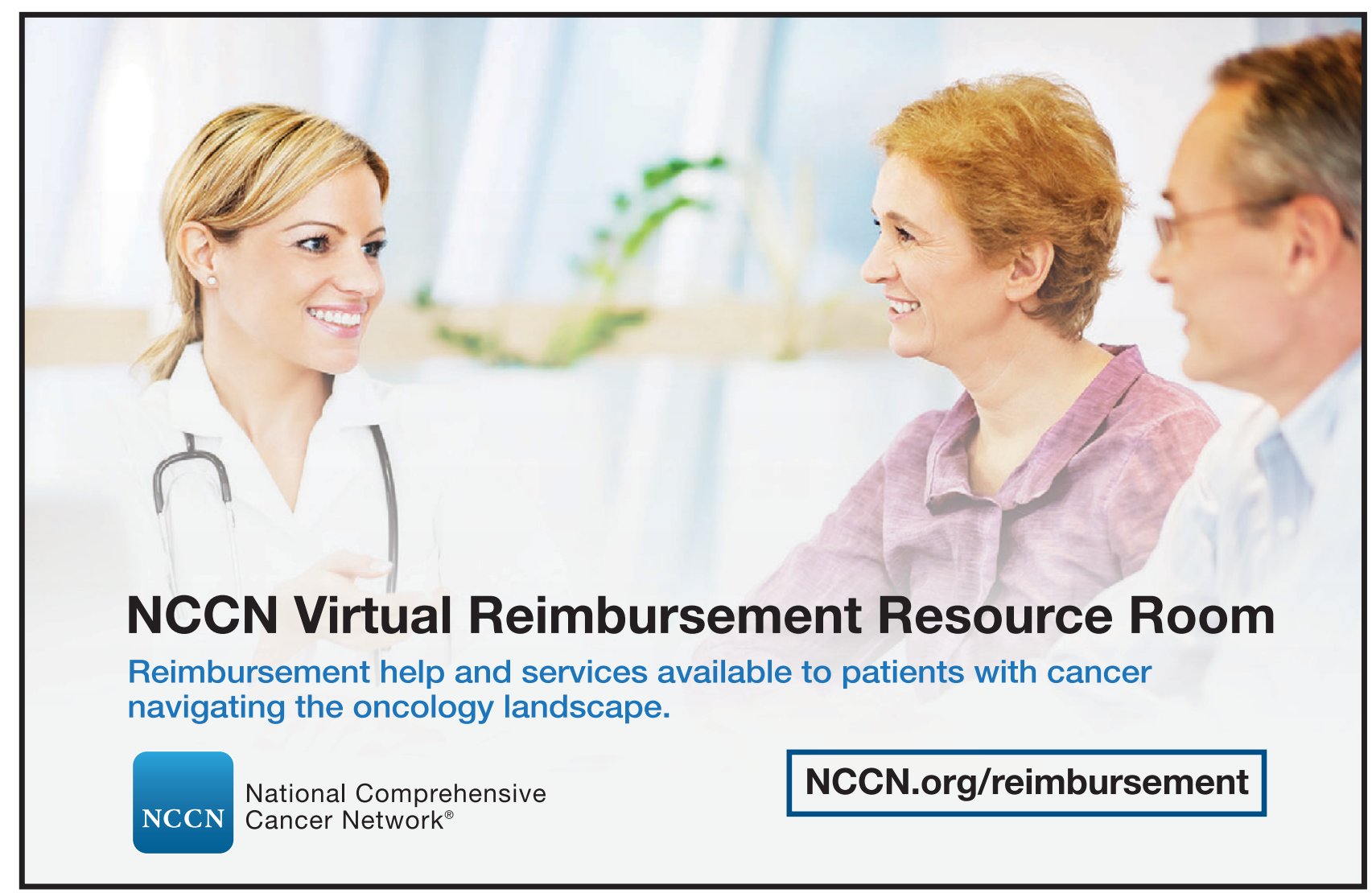

